Verification of the Effectiveness of the Onl i ne Tuni ng Systemfor Unknown Person in the Awaki ng Behavi or Detecti on System

\begin{tabular}{|l|l|}
\hline 著者 & Sat oh H r onobu, Takeda Fumiaki \\
\hline $\begin{array}{l}\text { j our nal or } \\
\text { publ i cat i on t i t l e }\end{array}$ & Lect ure Not es i n Comput er Sci ence \\
\hline vol une & 5518 \\
\hline page range & $272-279$ \\
\hline year & 2009-06-06 \\
\hline URL & ht t p: //hdl . handl e. net /10173/614 \\
\hline
\end{tabular}




\title{
Verification of the Effectiveness of the Online Tuning System for Unknown Person in the Awaking Behavior Detection System
}

\author{
Hironobu Satoh and Fumiaki Takeda \\ Kochi University of Technology \\ 185, Miyanokuchi, Tosayamada-cho, Kami-shi, Japan \\ \{satoh.hironobu, takeda.fumiaki\}@kochi-tech.ac.jp \\ http://www.kochi-tech.ac.jp
}

\begin{abstract}
We have developed an awaking behavior detection system using a neural network (abbreviated as NN). However, the detection ability of unknown people is not sufficient with compared to that of learned people. In this research, to improve the detection ability of unknown people, we apply an online tuning system using a continuous learning of the $\mathrm{NN}$ for the detection system. In the online tuning system, only a few additional data of a new objective person are used for the continuous learning, where the weights of the NN converged in the initial learning are used as the initial weights for the continuous learning. In this paper, to verify an ability of the online tuning system, we compare detection ability of the converged initial learning with that of the converged online tuning.
\end{abstract}

Key words: Online tuning, Neural network, Awaking behavior detection system, Continuous learning

\section{Introduction}

Recently, accidents such that seniors fall down from the bed in care facilities or hospitals are increased[1,2]. To prevent a person from falling of a bed, we have developed an awaking behavior detection system[3-6] using a neural network[7] (abbreviated as NN) and a captured picture by a WEB camera. However, the detection ability of the unknown people is not sufficient with compared to that of learned data. And, for the detection using weights of the NN, learning data of which is sampled from some people, do not had enough detection ability of the unknown people. Moreover, a few picture of the subject for the detection can be captured. In this research, to improve the detection ability of the unknown data, we apply an online tuning system[8] using a continuous learning of the NN to the awaking behavior detection system. In the online tuning system, only a 
few additional data of the new objective person for the detection are used for the continuous learning, where the weights of the NN converged in the initial learning are used as the initial weights for the continuous learning. By the online tuning system, the detection ability of the awaking behavior detection system is customized in the new objective person's data. In this paper, to verify an ability of the online tuning system, we compare the detection ability of the converged initial learning with that of the converged online tuning.

\section{System Construction}

\subsection{Hardware}

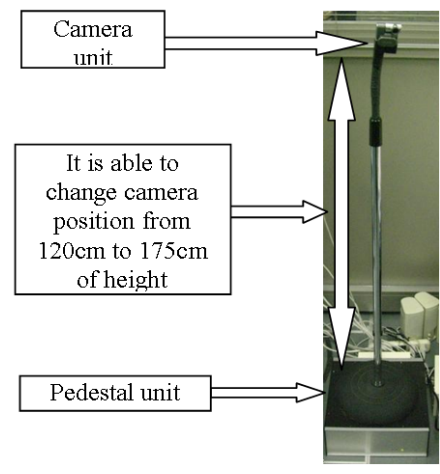

Fig. 1. The awaking detection apparatus.

The awaking detection apparatus is shown in Fig. 1. It is consists of a camera unit and a pedestal unit. The Web camera is set in the camera unit. It can be adjusted from $120 \mathrm{~cm}$ to $175 \mathrm{~cm}$ above the floor. The pedestal unit, which includes a personal computer, must be as near as possible so that it can be set freely around the bed. As shown in Fig. 2, the apparatus is set beside the bed. In Fig.2, the upper drawing line from the WEB camera shows the upper limit of the sight of the detection system.And, the lower one shows a lower limit of the sight. 


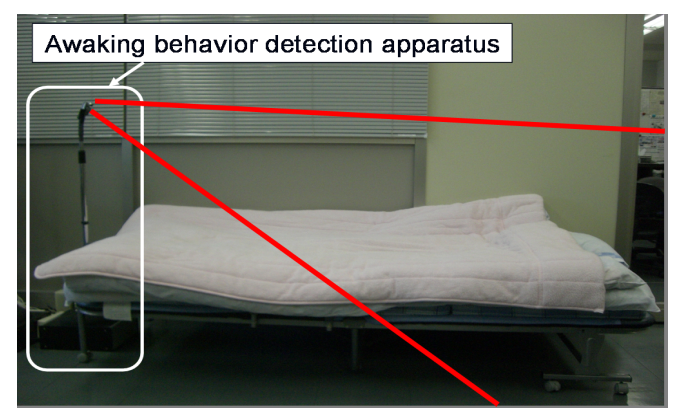

Fig. 2. The awaking behavior detection apparatus and a bed.

\subsection{Software}

The awaking behavior detection system classifies the objective person's behavior into five states. Variety of the awaking states is shown in Fig. 3.

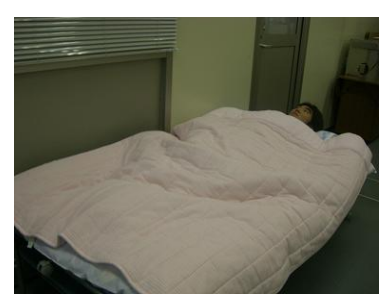

(a) Lying in the bed

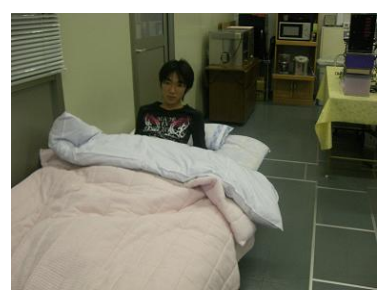

(b) Being sitting up in the bed

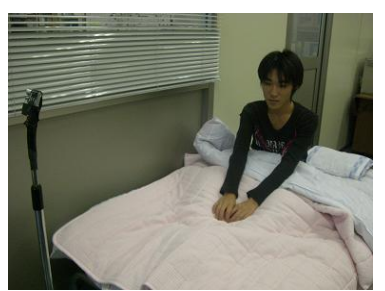

(c) Sitting in the bed

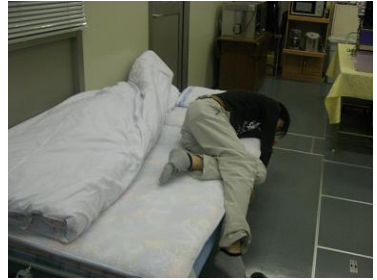

(d) Almost falling from the bed

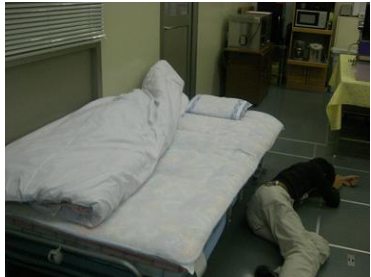

(e) Having fallen from the bed

Fig. 3. The variety of the awaking states 
In this system, five captured pictures, which are captured by the Web camera, are used for once recognition of the objective person's behavior. Each pictures is captured every one second. To located the objective person's face in the center of the picture, the objective person's figure is clipped from the captured picture and saved. Four pictures among captured five pictures are used as dynamic information. Another picture is used as static information. Five pictures are defined one data set for the detection or the learning. In the first place, the feature extraction method of the dynamic information is shown. First, four pictures are transformed the gray-scale pictures using the following equation[9].

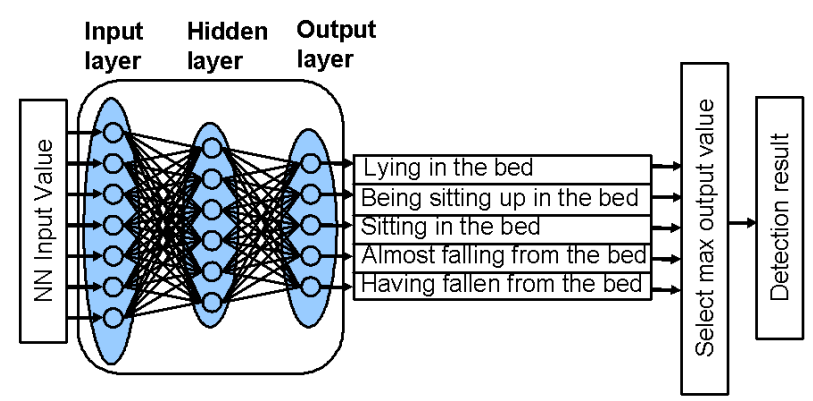

Fig. 4. The construction of the NN.

$$
V=(R \times 30+G \times 59+B \times 11) / 100
$$

Where $\mathrm{V}$ means transformed after brightness value, $\mathrm{R}$ means red brightness value, $G$ means green brightness value, $B$ means blue brightness value. The gray-scale pictures are divided into $36(6 \times 6)$ blocks. Then, each block's values are averaged on each line and each column. Next, averaged values are binarized using a threshold. Finally, these values are input to the NN. In the second, the feature extraction method of the static information is shown. First, the picture is transformed the gray-scale pictures using equation (1). The gray-scale picture is divided into $200(20 \times 10)$ blocks. Then, each block's values are averaged. Next, averaged values are binarized using a threshold. Finally, input these values into the NN.

The NN as shown in Fig.4, consists of an input layer with 200 neurons, a hidden layer with 30 neurons, and an output layer with five neurons, where output neuron corresponds to state of the objective person's behavior. We use the improved back propagation algorithm equation as a learning algorithm[10].

$$
\Delta W_{j k}(t)=-\eta \delta_{k} O_{j}+\alpha \Delta W_{j k}(t-1)+\beta \Delta W_{j k}(t-2)
$$

Where $\Delta \mathrm{W}$ means the amount of the correction of the weight, $\mathrm{t}$ means the sample number, $\eta$ means the positive learning coefficient, $\alpha$ means the proportional coefficient of the inertia term, and $\beta$ means the proportional coefficient 
of the oscillation term. This algorithm makes it possible to converge faster than usual back propagation algorithms, and escape easier than that one from a local minima.

\section{Online Tuning}

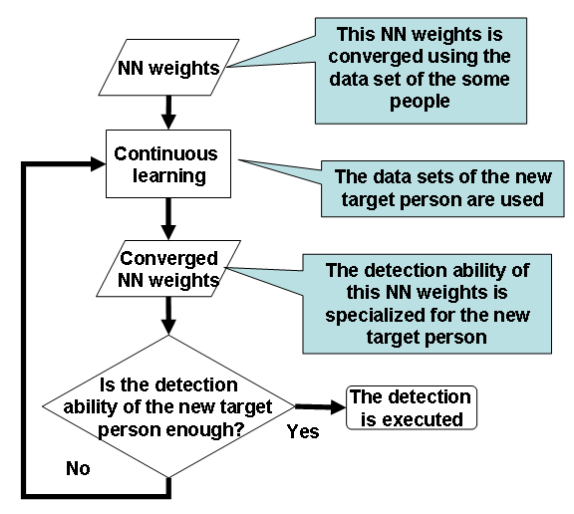

Fig. 5. The online tuning procedure.

The purpose of the online tuning is to adapt the detection ability of the awaking detection system to the new objective person using a few additional data. Fig. 5 shows the online tuning procedure. In the awaking behavior detection system, that's procedure is shown as follows. First, the behaviors of some people are captured. Then, initial learning is executed using the captured data sets. Next, the behaviors of the new objective person is captured for the online tuning. Then, the continuous learning is executed using captured data set of the new objective person, where the weights of the NN converged in the initial learning are used as the initial weights for the continuous learning. And the continuous learning is repeated, until user is satisfied with the detection ability of the new objective person.

We consider that the detection ability using the online tuning is higher than that of the initial learning only using the captured data of the new objective person. The detection ability of the awaking detection system is adapt to a new objective person. And, that of the awaking detection system is specialized in the new objective person.

\section{Experiment}

In this section, we show the NN weights, learning data which is sampled from some people, have not enough detection ability of the unknown person's data. 
Moreover, demonstrating the effectiveness of the online tuning, we verify that the detection ability of the online tuning is higher than that of the initial learning using the captured data of the new objective person for detection.

The experiment method is shown as follows. First, five awaking states of the subject are captured using the WEB camera. The subject is four male (Subject A, Subject B, Subject C, Subject D). The subjects wears two kinds of clothes. One clothes is black clothes. Another is white one. Then, the figure of the subject is extracted from captured picture by manual.

As the experiment process for verifying the detection ability of the online tuning, first, three subjects are chooses from four subjects. Next, three data sets per pattern and per subjects are chosen from three chosen subjects. The chosen data sets are used for the initial learning of the online tuning. Then, targeted data set, which is chosen from the other subject, are used for the online tuning.

As the experiment process for verifying the detection ability of the initial learning, The targeted data sets, which is same used data sets in online tuning, are used for the initial learning.

A detection data set is used for verifying of the detection ability using online tuning and that of the initial learning after the online tuning or the initial learning are converged. The number of the detection data set is 60 . The comparing the detection ability of the online tuning with that of the initial learning is repeated several times modified the number of the targeted data set.

Table 1. The detection rate when the initial learning of the online tuning is converged. The gray color of the cell where the detection rate of the unknown subject is shown.

\begin{tabular}{|c|c|c|c|c|}
\hline $\begin{array}{c}\text { The detection } \\
\text { data set }\end{array}$ & $\begin{array}{c}\text { Subject A } \\
\text { (success/ }\end{array}$ & $\begin{array}{c}\text { Subject B } \\
\text { (success/ } \\
\text { population) }\end{array}$ & $\begin{array}{c}\text { Subject C } \\
\text { (success/ } \\
\text { population) }\end{array}$ & $\begin{array}{c}\text { Subject D } \\
\text { population) } \\
\text { (success/ } \\
\text { population) }\end{array}$ \\
\hline $\begin{array}{c}\text { Subject B, Subject C } \\
\text { and Subject D }\end{array}$ & $\begin{array}{c}78.66 \% \\
(236 / 300)\end{array}$ & $\begin{array}{c}98.33 \% \\
(295 / 300)\end{array}$ & $\begin{array}{c}95.33 \% \\
(286 / 300)\end{array}$ & $\begin{array}{c}95.66 \% \\
(287 / 300)\end{array}$ \\
\hline Subject A, Subject C & $100.00 \%$ & $58.00 \%$ & $96.33 \%$ & $95.00 \%$ \\
and Subject D & $(300 / 300)$ & $(174 / 300)$ & $(289 / 300)$ & $(285 / 300)$ \\
\hline Subject A, Subject B & $98.00 \%$ & $98.66 \%$ & $78.66 \%$ & $98.00 \%$ \\
and Subject D & $(294 / 300)$ & $(296 / 300)$ & $(236 / 300)$ & $(294 / 300)$ \\
\hline Subject A, Subject B & $96.33 \%$ & $98.00 \%$ & $94.33 \%$ & $68.66 \%$ \\
and Subject C & $(289 / 300)$ & $(294 / 300)$ & $(283 / 300)$ & $(206 / 300)$ \\
\hline
\end{tabular}




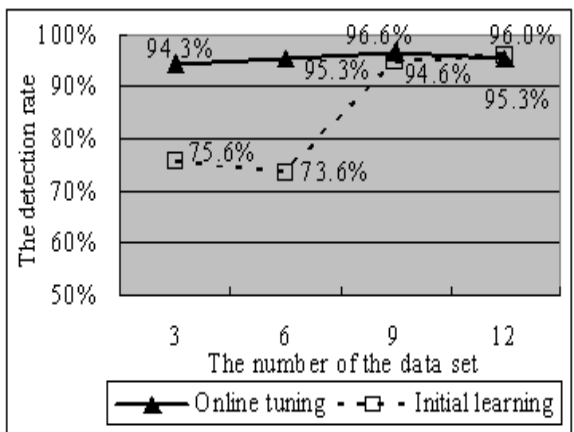

(a) The new objective person is Subject $A$.

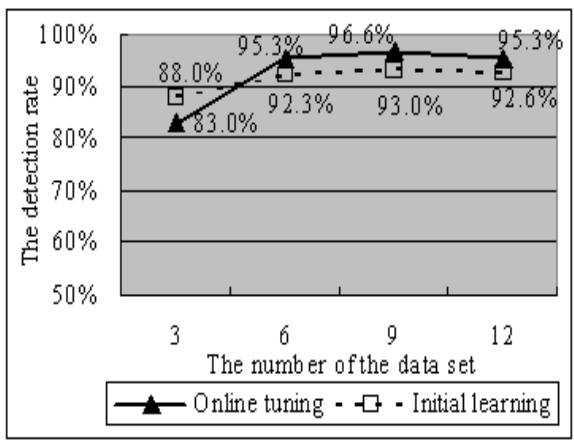

(c) The new objective person is Subject $\mathrm{C}$.

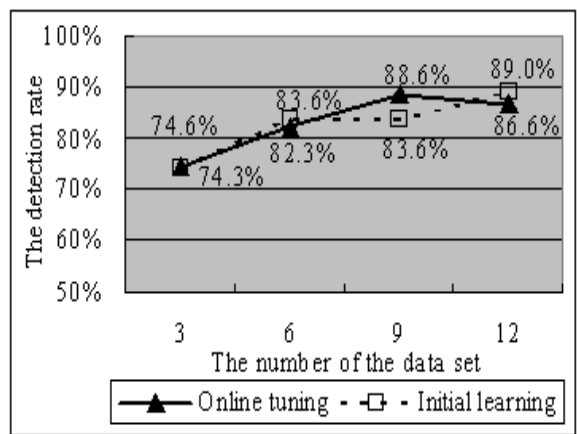

(b) The new objective person is Subject $B$.

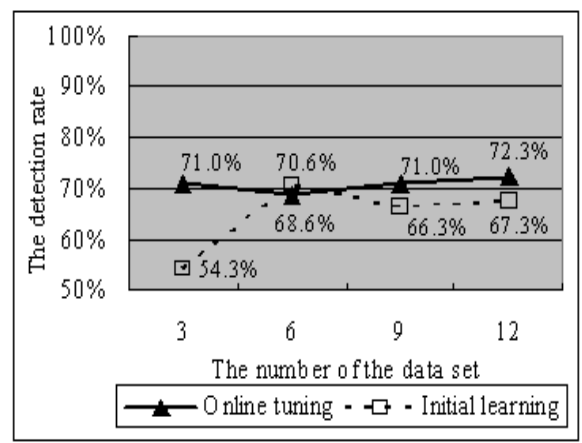

(d) The new objective person is Subject $\mathrm{D}$.

Fig. 6. Comparison of the detection ability.

Table.1 shows the detection rate of each subject when the initial learning of the online tuning is converged. Fig.6(a) shows the results of the comparing the detection ability of the online tuning with that of the initial learning, when the targeted subject is Subject A. Fig.6(b), Fig.6(b) and Fig.6(b) also show the results of the comparing the detection ability of the converged online tuning with that of the converged initial learning, when the objective subject is Subject B, Subject $\mathrm{C}$ and Subject D. In those figures, the vertical axis indicates the average of the detection rate of five subject's behaviors. Horizontal axis indicates the number of the data set of the new objective subject.

From the experiment results, since the detection rate of the unknown subject is lower than that of the learned subject, the detection ability of the unknown 
subject is not effective when the $\mathrm{NN}$ is converged using the data set of some people. Moreover, we verified the detection ability of the converged online tuning is higher than that of converged the initial learning. This result has a probability of $68.75 \%$ (The number of the effectiveness of the online tuning than that of the initila learning is eleven. The number of the effectiveness of the initial learning than that of the online tuning is five.). When nine targeted data sets are used, the detection ability of converged the online tuning in all objective subujects is higher than that of the converged initial learning.

\section{Conclusion}

It can concluded that the detection ability of unknown person was not enough, from the comparison the detection rate of the unknown subject with that of the learned subject. When the nine targeted data sets is used for the online tuning, the effectiveness of the online tuning system is demonstrated. In addition, by using the online tuning, the detection ability of the awaking behavior detection system is useful for customizing for the unknown person.

\section{References}

1. Mogi, R., Ishii, T., Suwa, M.: Present state and analysis of tipping over fall. Tokyo metropolitan government public health academy journal No.101, 184-185 (1998)

2. Yamazaki, T., Sugawara, Y., Hoshino, M., Ozaki, F.: Risk Factors Related to Falls from Beds and a Study on Preventing Patient's Fall. Journal of the Japan Society of Nursing Vol.12, No.1, pp.2-12 (2003)

3. Ikeda, R., Satoh, H., and Takeda, F.: Development of Awaking Behavior Detection System Nursing Inside the House. In: International Conference on Intelligent Technology 2006, Taipei, pp.65-70 (2006)

4. Matubara, T., Satoh H., and Takeda, F.: Proposal of an Awaking Detection System Adopting Neural Network in Hospital Use. In: World Automation Congress 2008, Hawaii, CD-ROM (2008)

5. Satoh, H., Takeda, F., Shiraishi, Y., Ikeda, R.: Development of a Awaking Behavior Detection System Using a Neural Network. IEEJ Trans. EIS, Vol.128, No.11, pp. 1649-1656 (2008)

6. Yamanaka, N., Satoh, H., Shiraishi, Y., Matsubara, T., Takeda, F.: Proposal of The Awakening Detection System Using Neural Network and It's Verification. In: The 52nd The institute of Systems, Control and information Engineers, Kyoto, CD-ROM (2008)

7. Haykin, S.: Neural Networks a comprehensive foundation. New jersey, USA, pp.161$173(1998)$

8. Satoh, H., Takeda, F.: Development of DSP unit for Online Tuning and Application to Neural Pattern Recognition System. In: 8th International Conference on Knowledge-Based Intelligence Information \& Engineering Systems,

9. Suematsu, Y., Yamada, H.: Image Processing Engineering. Tokyo, Japan, pp.22-23 (2000)

10. Takeda, F.,Omatu, S., Inoue, T.,Onami, S.: Bill Money Recognition Using Neural Network with FFT as Pre-processor. Transactions of the Institute of Systems, Control and Information Engineers. Vol.5, No.7, pp.9-17 (1992) 